\title{
Development and efficacy of feed-based recombinant vaccine encoding the cell wall surface anchor family protein of Streptococcus agalactiae against streptococcosis in Oreochromis sp.
}

\begin{abstract}
This study was carried out to determine the antibody responses and protective capacity of an inactivated recombinant vaccine expressing the cell wall surface anchor family protein of Streptococcus agalactiae following oral vaccination against streptococcosis in tilapia. Tilapia were vaccinated orally with $106 \mathrm{CFU} / \mathrm{mL}$ of the recombinant vaccine incorporated in feed (feed-based recombinant vaccine) (vaccinated group or Group 1), $106 \mathrm{CFU} / \mathrm{mL}$ of $\mathrm{pET}-32$ Ek/LIC vector without cell wall surface anchor family protein (control group or Group 2), $106 \mathrm{CFU} / \mathrm{mL}$ of formalin-killed cells of S. agalactiae vaccine incorporated in feed was also prepared (feed-based vaccine) (vaccinated group or Group 3), and unvaccinated control group or Group 4 (fed with commercial pellets). During the course of study, serum, mucus and gut lavage fluid were collected to evaluate the antibody levels via enzyme-linked immunosorbent assay (ELISA). The results showed that tilapia immunized with the feed-based recombinant vaccine developed a strong and significantly $(\mathrm{P}<0.05)$ higher $\operatorname{IgM}$ antibody response in serum, mucus and gut lavage fluid samples compared to groups 2, 3 and 4 . Following heat intervenes and intraperitoneal challenge, the rate of survivors (RPS) was $70 \%$ for the vaccinated group, and $0 \%$ for the rest of the groups. Therefore, the study revealed that the feed-based recombinant vaccine significantly provides high protection against high dose challenge in heat stress environment and enhances the production of the mucosal and humoral immunity.
\end{abstract}

Keyword: Recombinant; Outer surface anchor family protein; Streptococcus agalactiae; Red hybrid tilapia 